\title{
Research on animation modeling and 3D capture technology based on affective computing
}

\author{
FenghuaYe \\ Guangdong University of Technology ,Guangzhou , 510075,China
}

Key words: affective computing; animation modeling; 3D rendering; motion capture; new media

\begin{abstract}
Animation is endowed with a lot of emotions and thoughts as an artistic form with independent aesthetic meaning, and it requires the creation of emotional methods how to properly handle the various emotion in the animation design together. The emotion animation modeling and 3D capture technology had been presented in this study, from the perspective of new media art, and based on the affective computing method, and its feasibility has been verified through the analysis, modeling and identification of animation emotion model. It can be concluded that animation emotional computing technology can successfully identify facial expression muscle contraction by animation Emotion Modeling and 3D expression capture experiment, which provide a useful data for facial expression animation rendering and manufacturing.
\end{abstract}

\section{Introduction}

The basic principles of animation are visual principles as well as film and television. In recent years, the new vitality of the animation industry has been brought by the development of computer science, making animation more exciting, and better visual effects. But no matter how the animation design technology changes, the idea that human animation always want to express the true feelings has not changed. If an animation is lack of thought and emotion, it will not give people a very good appreciation of the feelings even the best technology, the impressive animation has its unique style and emotional thoughts. This research lays the technical foundation for the deduction of the unique style and emotional artistic conception, mainly based on the principle of emotional computing, starting from the emotional modeling of animation, and capture the basic expression of 3D animation.

\section{Emotion creation method in animation design}

In the animation design process, in order to reflect the authenticity of the animation, emotional design in the process of animation design is one of the important factors to be considered, there are mainly four animation creation methods, the following are described in detail in this paper.

(1) Grasp the emotional environment of animation

The role model and environment of animation are complementary, forming a whole emotional environment in the process of deduction. Animation film mainly to the audience to show the role of animation characters and ideas, and these content and ideas need to obtain from the overall environment and macro consciousness. The creation of animation emotional environment includes animation plot, scene, language, color, lighting, action, music, sound, etc. these environments provide the basic environment for the emotional expression of animation characters.

(2) Emotional creation of animation theme pluralism

Modern technology makes people's world outlook and the outlook on life has changed greatly, emotion for the animation has become diversified, so multiple themes must be realized in the emotion design and creation process of animation, so that it can accord with the appreciation level of the people. The change of modern science and technology to social environment has provided a more wide space for the designers of animation, animation design emotion will be bound to diversify, and the modern design must be embedded in affective computing.

(3) Symbolic creativity of emotional artistic conception

Emotional element is unable to measure in any way, its presence is between people and things of 
the mind in the animation design synaesthesia. When designing the emotional elements of animation, we need to express and calculate the emotion, and then convey the emotion to the audience through the visual and auditory materialized form. When the audience appreciates animation creation, the main reception way is accomplished by visual and auditory, animation design to achieve the perfect effect of emotion, the need for related affective computing, allowing more graphic symbols to achieve specific effects of emotional expressions.

(4) The form creation guided by animation visual effect

In the animation design, visual expression of emotion is mainly expressed by the form of animation, dynamic aesthetic effect will bring strong emotions in the animation of affective computing, the designer can of excellent animation action of capture, use a variety of creative means, with favorable space diversity to enhance the animation creation. In the actual design, if the 3D capture animation effect can be reasonable collocated, exquisite visual effect can be got, so that animation works becomes more emotional and glorious.

\section{Animation modeling and 3D capture technology based on affective computing}

Nine gold movement law: 1 (T\&S), Time control and Sense; 2, (C\&E) Compression and Extension; 3, (P) Planned action; 4, (K\& C1) Key action and Continuous action; 5, (F\&O) 6, Follow and Overlap; (SI\&SO) Slow in and Slow out 7, (P1) Performance; 8, (EX) Exaggeration; 9 (SA), the second Animation law, quantitative research can be carried out by taking the innovative essence of animation art, the "nine gold movement law", that can give the soul and emotion to animation character, as the affective computing signal and the measured variables.

In order to achieve affective computing in animation creation, modeling operations for emotional animation is needed, accurate motion capture technology to model reasonable calculation, so as to realize the self emotional expression of the creation of the animation. In order to achieve the objectivity of research and easy to achieve the purpose of this study mainly defines the four basic emotions, are happy and sad, love and hate, these emotions are composed of basic emotion, can realize the emotional self expression effect, and the emotional dimension based on a two-dimensional emotion space, specific the model shown in figure 1.

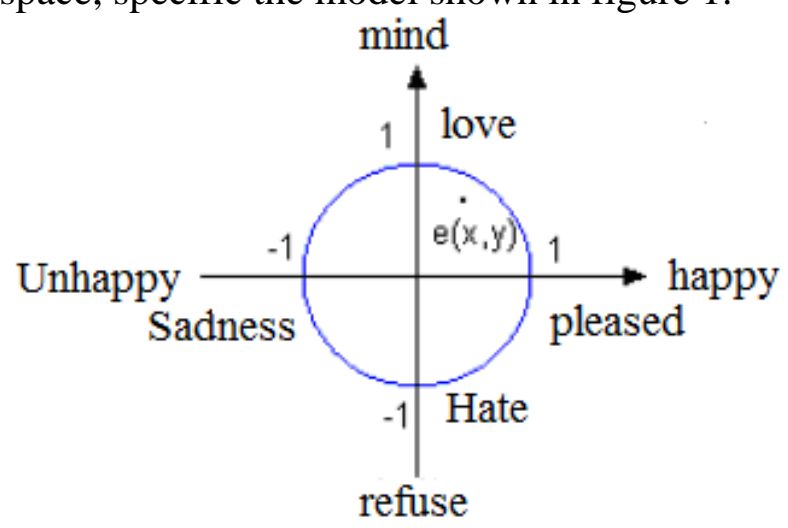

Fig. 1 The two-dimensional model of affective computing

As shown in Figure 1, two-dimensional composition of emotion space are happy and unhappy, mind and refuse respectively, almost all emotional expression can be found the corresponding points in round that the radius is 1 , which takes happy and unhappy as horizontal axis, and takes mind and refuse as the longitudinal axis. In the coordinate area, pleased and sadness are of the opposite sentiment, which is expressed as 1 and -1 in horizontal coordinate. Similarly, love and hate is expressed as 1 and -1 in the Vertical coordinate. Assuming a two-dimensional emotion vector (x, $y)$, the intensity of emotion is expressed as the norm of vector, the magnitude of the emotion reaching to which basic emotions can be impressed as (x,y), then it can be got that, 


$$
\begin{aligned}
& Y=\sqrt{x^{2}+y^{2}} \\
& \theta=\left\{\begin{array}{lll}
\arccos \left(x / \sqrt{x^{2}+y^{2}}\right), & \text { when } & y>0 \\
\pi+\arccos \left(x / \sqrt{x^{2}+y^{2}}\right), \text { when } & y<0
\end{array}\right.
\end{aligned}
$$

In affective computing process, if the emotional points are evenly distributed, the emotion of the animation is not highlighted, if the emotional points are unevenly distributed, it can exhibit irritability, depression and other emotional feelings. Therefore in the animation emotion creation, according to the emotion computation principle and method, the animation emotion can be reconstructed through the animation expression capturing. the expression feature matrix extraction principle can be used in the animation expression capturing, the detailed process of the expression extraction is shown as follows,

(1) Select $n$ animation emotion action expression as feature matrix $Y=\left[y_{1}, y_{2}, y_{3}, \ldots, y_{\mathrm{n}}\right]$;

(2) Reduce the dimension of $Y$ by the following steps:

a. Getting the average value of emotion characteristic signal:

$$
\alpha=\frac{1}{n} \sum_{j=1}^{n} Y_{j}
$$

b. Solving covariance matrix:

$$
M=\frac{1}{n} \sum_{j=1}^{n}\left(Y_{j}-\alpha\right)\left(Y_{j}-\alpha\right)^{T}
$$

c. Determining the eigenvalues

$$
M r_{j}=\lambda r_{j}
$$

$d$. According to the detection precision $p$ to solve the first $a$ maximum eigenvalues

$$
\sum_{j=1}^{a} \lambda_{j} / n \sum_{j=1}^{a} \lambda_{j} \geq P
$$

$e$. Calculating the characteristic value of animation emotion and corresponding eigenvector $T$.

According to the extraction of emotional eigenvalue, we can get some performance characteristics of animation, such as animation action, facial expression and so on.

\section{Animation Emotion Modeling and 3D expression capture experiment}

In the modeling of animation emotion, simulation for complex behavior animation role is not just related to show through action, but also to the animation of the surrounding environment, according to the specific environment of animation role in the establishment of a role model, its principle is shown in Fig.2.

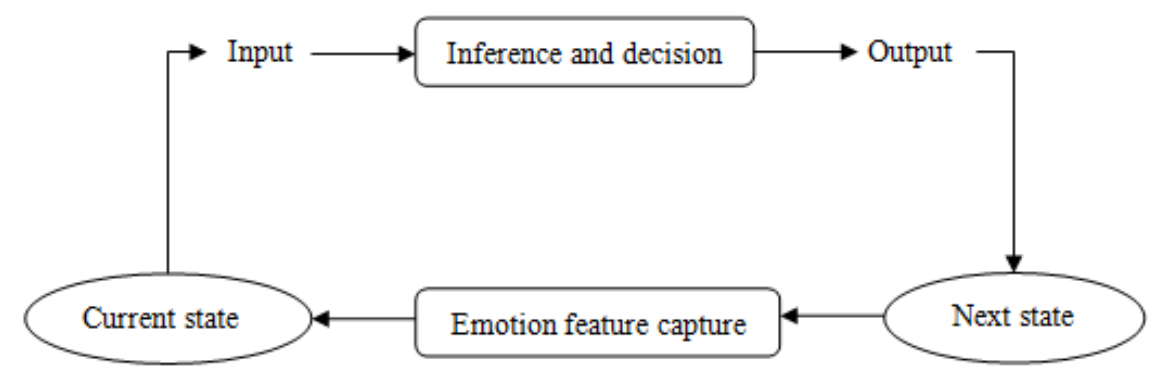

Fig. 2: The animation emotional role modeling schematic

As shown in Figure 2, the emotional modeling of animation characters is mainly the acquisition of information. By acquiring information, we can make decision and reasoning about its emotion characteristic expression, and get the next action expression state. Therefore, animation characters can be created based on state transition rules, as long as the role of the set of state features, through emotional computing, the creative process is as follows: 


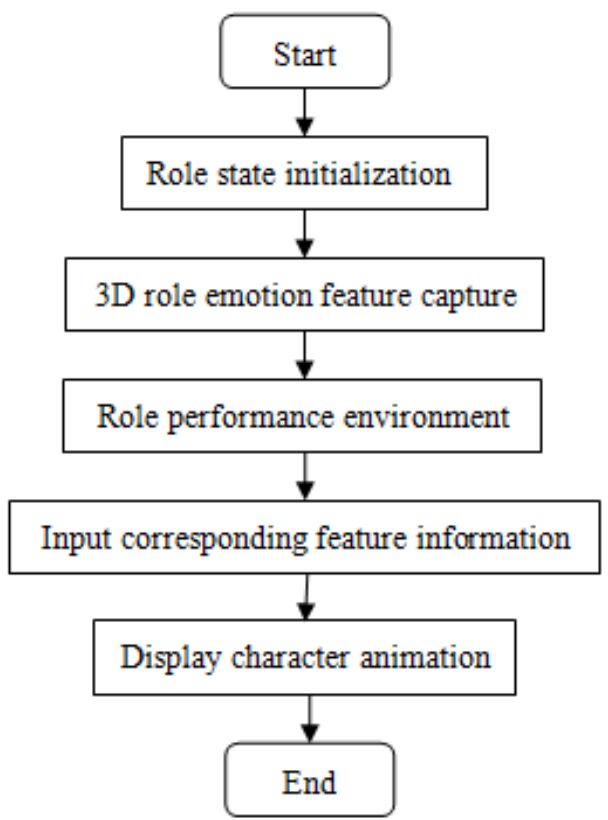

Fig.3: Animation emotional character creation process

As shown in Figure 3, in the animation role emotion in the process of creation, first initialize the role model, then the program environment initialization, using 3D capture technology environment and emotional expression, emotional feature information input the corresponding display, character animation. The corresponding emotion characteristic information of the input link, need to use 3D capture technology, on the emotional expression of characteristic signal capture, the study of emotional facial expressions happy 3D capture, has been shown in Table 1 shows the statistical results.

Table 1: Happy emotional expression muscle feature capture

\begin{tabular}{|c|c|c|c|c|c|}
\hline Happy emotion symbol & No expression & $20 \%$ & $40 \%$ & $60 \%$ & $80 \%$ \\
\hline $\begin{array}{c}\text { Mouth pressure } \\
\text { contraction of muscle } 1\end{array}$ & 0 & 0 & 0 & 0 & 0 \\
\hline $\begin{array}{l}\text { The amount of muscle } \\
\text { contraction } 2\end{array}$ & 0 & 0.21 & 0.42 & 0.63 & 0.85 \\
\hline $\begin{array}{c}\text { The amount of the main } \\
\text { muscle contraction } 3\end{array}$ & 0 & 0.18 & 0.31 & 0.49 & 0.68 \\
\hline $\begin{array}{l}\text { Nasal lip muscle } \\
\text { contraction } 4\end{array}$ & 0 & 0 & 0 & 0 & 0 \\
\hline
\end{tabular}

Table 1 shows the characteristic signal of 3D facial expression muscle happy emotion capture results, by capturing the results can be seen, the use of 3D capture technology can get emotional characteristic signal accurately, the data base for affective computing.

Table 2: Emotional facial expression statistics

\begin{tabular}{cll}
\hline Emotion type & AU & \multicolumn{1}{c}{ Feature description } \\
& parameter & \\
\hline Happy & AU3, 7,9 & Corners of the mouth, eyelids, mouth \\
Sad & AU1, 4,8 & Eyelids, lips, nose \\
surprise & AU6, 3,5 & Eyelid, nose, corners of the mouth \\
Hate & AU1, 8,2 & Eyelids, eyebrows, nose
\end{tabular}

As shown in Table 2, according to the process of animation creation of emotion, the emotion vector calculated emotional space, and then refer to the FACS system, to determine the AU expression of a series of parameters, the facial expression is determined by these parameters, the expression of emotion in the animation design can be directly used.

\section{Conclusions}

An emotion computing method has been described in this paper, emotion vector can be 
calculated by the emotional space, the characteristic emotion can be represented by the mode and angle of the emotion vector. The contraction of facial muscles is obtained by the 3D facial expression capture of happy mood, emotion vector is calculated in the mood space, and then a series of AU expression parameters is determined using the FACS system, which provides reference data for the design of animation emotion symbols expression. If animation design can achieve the perfect emotional effect, it is necessary to carry out the relevant emotional calculation, so that animation graphic symbols can achieve a specific emotional expression effect.

\section{References}

[1] Kong Xiaoli, Jiang Yuxiang, etc.. Simulation of curled aquatic plants based on Bezier curve. Journal of Suzhou University,31 (6), pp. 1-5, 2011.

[2] Hao Xiaozhong, AHMEDA.A.D, Chen Wenliang. Research and implementation of Bezier curved surface modeling method. China manufacturing informatization, 40 (23), pp. 42-45, 2011.

[3] Lv Liangfu, Zhang Jiawan, Sun Jizhou etc.. Review on visualization of network security. Journal of computer Applications, 28 (8), pp. 1924-1925, 2008.

[4] Liang Yun. Data mining algorithms and applications, Shen Chengfeng, Peking University press, Beijing, 2006.

[5] Yu Xiaosheng. Dimensionality reduction methods for high-dimensional data. Information Science. 25 (8), pp. 1248-1251, 2007.

[6] Sun Qindong. Detection of distributed denial of service attacks based on time series analysis. Chinese Journal of Computers, 28(5), pp.768-773, 2005.

[7] Hong Fei, Wu Zhimei. Adaptive estimation method of Hurst Exponent Based on wavelet. Journal of Software, 16 (9), pp. 1685-1689, 2005.

[8] Li Yongli, Liu Guizhong, Wang Haijun. Analysis of Hurst parameters of self similar data stream based on wavelet. Journal of Electronic \& Information Technology, 25 (1), pp. 100-106, 2003.

[9] Lv Liangfu, Dai Hua, Sun Jizhou. The improved block Davidson method for Solving Large Symmetric Eigenvalue. Journal of Tianjin University, 40 (5), pp. 559-562, 2007.

[10] Wu Wenguo, Jin Xiaogang, Feng Jieqing. Triangle morphing. Journal of computer-aided Design \& Graphics, 17 (7), pp.1615-1619, 2005.

[11] Su Shaoyong, Chen Jiming, Pan Jingui. Research on behavior modeling technology in virtual environment. Computer Science, 34 (2), pp.270-277, 2007.

[12] Wang Yiqun, Huang Cheng. Implementation and manipulation of 3DS file with object oriented method. Journal of Yancheng Institute Of Technology, 18 (3), pp. 41, 2005

[13] Meng Xiuyan, Wang Zhiliang, Wang Lijuan. Research on teaching aid system based on Emotion Modeling. Application Research of Computer, 24 (4), pp. 75, 2007

[14] Fang Yu, Chen Jiang, Yuan Zhuzhi. Emotional model building based on Artificial Intelligence, Information and Control, 35 (6), pp. 676, 2006 